\title{
Antibiotics Resistance Profile of Uropathogens Isolated from Al Buraimi Hospital, Sultanate of Oman
}

\author{
Mansoor Khalid ${ }^{1}$, Maryam Khalifa Al Naimi ${ }^{1,}$ Hafiz Ghulam Murtaza Saleem², \\ Muhammad Muqeet Ullah ${ }^{3} \&$ Ahmed Yar Mohammed Dawood Al Baloshi ${ }^{3}$ \\ ${ }^{1}$ Department of Laboratory Services, Al- Buraimi Hospital, Al Buraimi Governorate - Ministry of Health, Oman \\ ${ }^{2}$ University Institute of Medical Laboratory Technology, Faculty of Allied Health Sciences, University of Lahore \\ Pakistan \\ ${ }^{3}$ Directorate of Communicable Disease Surveillance and Control, DGHS, MOH Al Buraimi Governorate, Oman \\ Correspondence: Mansoor Khalid, Department of Laboratory Services, Al- Buraimi Hospital, Al Buraimi \\ Governorate - Ministry of Health, Oman. E-mail: mansoor.khalid111333@gmail.com
}

Received: October 19, 2017 Accepted: November 20, 2017 Online Published: December 1, 2017

doi:10.5539/gjhs.v10n1p98 URL: https://doi.org/10.5539/gjhs.v10n1p98

\begin{abstract}
Background: Urinary tract infections (UTIs) is a worldwide problem of all age groups and gender. Emerging resistance to antibiotics making difficult in the choice of treatment and management of UTI cases. This study sought to determine the gender wise prevalence of common uropathogens from UTI patients and the resistance profile of uropathogens against commonly used antibiotics.

Method: This cross sectional study was conducted in Al Buraimi Hospital, Sultanate of Oman. The data of UTI patients visited hospital was analyzed for the isolation of uropathogens from positive urine culture and antimicrobial sensitivity test was performed by disc diffusion method. Descriptive statistics was used to analyze the data using SPSS 21.0 and Microsoft Excel.

Result: Total 4,480 urine samples were analyzed for isolation of uropathogens and significant bacteriuria were found in total $846(19 \%)$ samples. Overall $728(86 \%)$ Gram-negative and $118(14 \%)$ Gram positive uropathogens were isolated from total (846) positive urine samples and the highest prevalence of isolates was observed in females $542(74 \%)$ than males $186(26 \%)$. E.coli was found the highest prevalent $(50.3 \%)$ uropathogens followed by Klebsiella species (13.9\%), Pseudomonas (6.3\%), A.baumannii (4\%), E.Cloacae (2.2\%), Proteus species (1.4\%), Citrobacter species (1.2\%), M. morgani (0.3\%) and Serratia species (0.1\%). The highest (34.3\%) antibiotic resistance was noticed in E.coli against Nalidixic Acid, however, susceptiblity was found against Ceftriaxone, Ceftazidime, Ciprofloxacin and Nitrofurantoin among female and males.

Conclusion: Overall, $\beta$ - lactam antibiotics, Cephalosporins, Fluroquiolones, Macrobids would be the first line of drugs and the most effective for the empirical treatment of Gram-negative and Gram-positive uropathogens; however Aminoglycosides, Carbapenems and Polymyxin could be used for the treatment of UTI infections as the second choice.
\end{abstract}

Keywords: uropathogens, resistance, antibiotics, MDRO (Multiple Drug- Resistant Organisms), UTI (Urinary Tract Infections)

\section{Introduction}

Urinary tract infections (UTI) fall into the category of either complicated or uncomplicated one and deem as one of the most common bacterial infections (Foxman, 2010). In most cases uncomplicated UTIs affects those individuals who are salubrious and do not exhibit any structural or neurological urinary tract irregularities (Hooton, 2012; Nielubowicz \& Mobley, 2010). These infections are divided into upper UTIs (pyelonephritis) and lower UTIs (cystitis) (Hannan et al., 2012; Hooton, 2012). Bacterial pathogens are perceived as the behind the cause of dispense symptoms, are treated with antibiotics ordinarily, in the case of primary care settings they account for nearly $95 \%$ of the antibiotic prescription for urinary tract infection (Ong, Kuyvenhoven, Van Dijk, \& Verheij, 2008). An estimation is $2 \%$ of boys and $8 \%$ of the girls face with no less than one episode of UTI by the age of seven years and recurrences occur in $12-30 \%$ of those cases within a year globally (Desai, Gilbert, \& McBride, 2016). Escherichia coli is conceded as the most frequent pathogen responsible for urinary tract infections among 
children (Hanna-Wakim et al., 2015). UTIs lead to substantial economic and public health burdens and significantly strike at life quality of affected individuals (Kostakioti, Hultgren, \& Hadjifrangiskou, 2012).

Uropathogens have unique characteristics, like they produce adhesins, toxins and siderophores that license them to colonize and seize the urinary tract, moreover they pass on between individuals both via person-to-person contact and very likely through water or food. While mainly self-limiting, treatment of UTIs with antibiotic therapy leads the way of an expeditious resolution of symptoms and more probably eliminate bacteremia. However, it also picks out commensal bacteria and resistant Uropathogens and cause the adverse effects on the vaginal and gut microbiota. Uropathogens are progressively becoming resistant to present time antibiotics, it could be the best time to investigate other possibilities to manage UTI (Foxman, 2010).

UTIs are caused by both Gram-positive and Gram-negative bacteria, in addition to fungi. The most frequent causative agent for both complicated and uncomplicated UTIs is uropathogenic Escherichia coli (UPEC). In the case of uncomplicated UTIs Klebsiella pneumoniae, Staphylococcus saprophyticus, Enterococcus faecalis, group B Streptococcus (GBS), Proteus mirabilis, Pseudomonas aeruginosa, Staphylococcus aureus and Candida spp joined after UPEC in prevalence (Foxman, 2014; Kline, Schwartz, Lewis, Hultgren, \& Lewis, 2011; Nielubowicz \& Mobley, 2010; Ronald, 2002). Following UPEC the order of prevalence for causative agents in complicated UTIs are Enterococcus spp., K. pneumoniae, Candida spp., S. aureus, P. mirabilis, P. aeruginosa and GBS (Chen, Ko, \& Hsueh, 2013; Fisher, Kavanagh, Sobel, Kauffman, \& Newman, 2011; Jacobsen, Stickler, Mobley, \& Shirtliff, 2008; Levison \& Kaye, 2013).

Patients suffer from a symptomatic UTI frequently treated with antibiotics; these treatments can cause long-term alteration in the normal micro-biota of the gastrointestinal tract and vagina, furthermore, in the burgeoning of multidrug-resistant microorganisms (Kostakioti et al., 2012). The availability of niches that are not anymore packed with the altered microbiota can intensify the risk of colonization with multidrug-resistant uropathogens. It is important that the 'golden era' of antibiotics is dwindling and therefore, the demand for rationally designed and alternative treatments is escalating (Hannan et al., 2012; Kostakioti et al., 2012) the members of the (Garau, 2008; K. Gupta \& Bhadelia, 2014; Pendleton, Gorman, \& Gilmore, 2013) lactamases (ESBLs). These plasmids abruptly proliferate resistance to third-generation cephalosporin besides other antibiotics (Chen et al., 2013; Garau, 2008; K Gupta \& Bhadelia, 2014; Paterson, 2006; Pendleton et al., 2013). Other members of the Enterobacteriaceae family result in the production of the class $\mathrm{C} \beta$-lactamases (AmpC enzymes) that are active against cephamycin as well as third generation cephalosporins, furthermore it is also resistant to $\beta$-lactamase inhibitors (Garau, 2008; K. Gupta \& Bhadelia, 2014; Paterson, 2006; Pendleton et al., 2013). The expression of AmpC enzymes is also coupled with carbapenem resistance in K. pneumoniae strains deficient of a $42 \mathrm{kDa}$ outer-membrane protein (Chen et al., 2013; Paterson, 2006). Multidrug resistance is also found among enterococci, while they are resistant to penicillin, cephalosporins, trimethoprim and clindamycin in a natural manner (Chen et al., 2013; K. Gupta \& Bhadelia, 2014; Pendleton et al., 2013). Currently Enterococcus spp. have exhibited high resistance to glycopeptides, together with vancomycin, which is believed to be the among last line of defence against multidrug-resistant organisms. Precisely, enterococci gradually develop resistance to glycopeptides through the expression of vancomycin and teicoplanin A-type resistance (van) genes that encode the penicillin-binding proteins (PBPs) VanA, VanB, VanD, VanE, VanG and VanL (K. Gupta \& Bhadelia, 2014; Pendleton et al., 2013). The mechanism of resistance for VanA, the most common PBP expressed by enterococci, is to take the place of the cell wall precursor D-alanineD-alanine with D-alanine-D-lactose, bring down the binding inclination towards vancomycin in an effective manner (Courvalin, 2006). The main objectives of the study were to identify the frequency, distribution, common uropathogens, antibiotics sensitivity and resistance and prevalent age group and gender suffering from urinary tract infection.

\section{Material and Methods}

\subsection{Study Design}

A retrospective study was conducted in Al- Buraimi Hospital, Al Buraimi Governorate - Ministry of Health, Oman. The electronic record (Al Shifa3+) data of suspected cases of UTI were obtained for urinary culture and sensitivity that visited hospital from January 2014 to December 2014. The samples were also referred from primary health care institutions (Buraimi Polyclinic, primary health institutes) and from Buraimi Hospital.

\subsection{Study Population}

The patients that visiting hospital with sign and symptoms and laboratory findings leading to urinary tract infections were included in this study. Study subjects were distributed into 4 different age groups to check the frequency of uropathogens among different ages. Group distribution of UTI patients was as follows: group I $(<12)$, 
group II (13 to 40 years), group III (41 to 60 years) and group IV (>60 years). The selection of patients was according to the following criteria:

\subsection{Inclusion Criteria}

The patients with sign and symptoms of UTI including suprapubic pain, urgency, frequency and dysuria, were selected for this study.

\subsection{Exclusion Criteria}

The patients who received antimicrobials treatment within 48 hours prior to entry were excluded from the study.

\subsection{Sample Size}

The number of samples in this study was 4,480 .

\subsection{Laboratory Analysis}

Mid-stream clean catch urine samples were collected in universal sterile containers $(20 \mathrm{ml})$. The samples were streak on Cysteine Lactose Electrolyte Deficient (CLED) (Oxoid, UK) and blood agar (Oxoid, UK) media with a standardized wire loop and were incubated overnight at $37^{\circ} \mathrm{C}$. Sterile calibrated wire loop of 0.001 liter (L) or 1 microliter $(\mu \mathrm{L})$ were used for colony count of urinary isolates. Bacterial growth was reflected as per Kass count or Kass criteria i.e. (single species count more than 105 organism/ml of urine) (Kass, 2002). Colonies were biochemically characterized according to the Borrows' guidelines (Barrow \& Feltham). Isolated colonies were further sub cultured on MacConkey (Oxoid, UK) and blood agar media (Oxoid, UK) to obtain the pure growth. Standardized identification (API 20 E, 20 NE and 20 Strip) system (Biomeriex, France) was used to identify and confirm the strain of isolates.

Antibiotic susceptibility testing of different uropathogens were determined by Kirby Bauer disk diffusion method (Boyle, Fancher, \& Ross, 1973). Muller Hinton Agar (MHA) agar media was used to evaluate the sensitivity and resistance pattern, pure bacterial colonies were spread on the MHA plates and were incubated for 24 hours at $35^{\circ} \mathrm{C}$ - $37{ }^{\circ} \mathrm{C}$. Zone of inhibition for bacterial growth were measured after incubation and compared as per CLSI guidelines (Orasch et al., 2014). Both Gram negative and positive isolates were tested for sensitivity against different groups of antibiotics. A total of 846 uropathogens were subjected for antibiotic sensitivity pattern against 24 different types of antibiotics as shown in Table 1.

Table 1. Drugs used in the study for antimicrobial sensitivity against Gram $(-/+)$ urinary isolates

\begin{tabular}{|c|c|}
\hline First Line & Second Line \\
\hline \multicolumn{2}{|l|}{ Augmentin (AMC30), Ciprofloxacin( CIP5), } \\
\hline Nitrofurantoin (F300),Nalidixic Acid (NA30), & Amikacin( AK30), Ceftazidime(CAZ30) Ceftriaxone( CRO 30), \\
\hline Cefotaxime (CTX30), Ampicillin (AMP & 25), Imipenem (IPM10), Cefotaxime (CTX30), \\
\hline \multirow[t]{4}{*}{ Gentamycin(CN10), Cotrimoxazole(SXT25) } & Meropenem (MEM10), Cefoxitin (FOX30), \\
\hline & Polymyxin (PB300),Vancomycin (VA30), \\
\hline & $\begin{array}{l}\text { Tecoplanin (TEC30), Linzolid( LZD30), piperacillin-Tazobactam } \\
\text { (TZP110), Colistin Sulphate (CT10), Penicillin( P10), }\end{array}$ \\
\hline & Oxacillin (OX1), Rifampicin (RD15) \\
\hline
\end{tabular}

Descriptive statistics was used to analyze the data after entering the data into SPSS 21.0 and Microsoft Excel in order to see the distribution and pattern of antibiotic resistance among uropathogens.

\section{Results}

In this study total of 4,480 urine samples were analyzed. Among them, 3903 from Buraimi Hospital and 577samples from Primary Health Care. Significant bacteriuria were found in total 846 (19\%) urine samples, out of those 745 (88\%) samples obtained from Buraimi Hospital and 101 (12\%) samples from Primary Health Centers, while remaining 3634 (81\%) urine samples were observed either non-significant bacteria, very low bacteria or sterile. Out of 846 urine samples of significant bacteriuria 629 (74\%) were female and 217 (26\%) were male. Overall 728 (86\%) Gram-negative and 118 (14\%) Gram positive uropathogens were isolated from total (846) positive urine samples. Out of 728 uropathogens $542(74 \%)$ and $186(26 \%)$ uropathogens were isolated from female and male patients respectively (Table 2). E.coli was found the highest prevalent $(50.3 \%)$ uropathogens in UTI 
followed by Klebsiella species (13.9\%), Pseudomonas (6.3\%), A.baumannii (4\%), E.Cloacae (2.2\%), Proteus species $(1.4 \%)$, Citrobacter species $(1.2 \%)$, M. morgani $(0.3 \%)$ and Serratia species $(0.1 \%)$. The Extended Spectrum $\beta$ Lactamase (ESBLs) producers pathogens were also isolated, the most prevalent were E. coli (16.6\%) followed by Klebsiella species (2.5\%) and Carbapenem Resistant Enterobacteriaceae (CRE) (Klebsiella) (0.1\%). Total 118 (14\%) Gram-positive uropathogens were isolated from urine samples among those 87 (74\%) uropathogens from female and 31(26\%) from male (Table 2). Group "D" Streptococci were the most frequent occurring uropathogens (54.2\%), followed by Group "B"Streptococci $(31.4 \%)$, S.aureus $(13.6 \%)$ and S. saprophyticus $(0.8 \%)$.

Table 2. Prevalence of uropathogens in UTI patients

\begin{tabular}{|c|c|c|c|c|}
\hline S. No & Uropathogens & Female $(\mathrm{n} / \%)$ & Male (n/\%) & Total \\
\hline \multicolumn{5}{|c|}{ (a) Gram Negative Bacilli } \\
\hline 1 & E. coli & $297(55)$ & $69(37.1)$ & $366(50.3)$ \\
\hline 2 & E.coli (ESBLs) & $79(15)$ & $42(22.6)$ & $121(16.6)$ \\
\hline 3 & Klebsiella species & $72(13)$ & $29(15.6)$ & $101(13.9)$ \\
\hline 4 & Klebsiella pneumoniae(ESBLs) & $7(1.3)$ & $11(6)$ & $18(2.5)$ \\
\hline 5 & Klebsiella pneumoniae(CRE) & $0(0)$ & $1(0.5)$ & $1(0.1)$ \\
\hline 6 & Pseudomonas species & $35(6.4)$ & $11(6)$ & $46(6.3)$ \\
\hline 7 & Pseudomonas aeroginosa (MDRO) & $2(0.4)$ & $3(1.6)$ & $5(0.7)$ \\
\hline 8 & Acinetobacter baumannii & $23(4.2)$ & $6(3.2)$ & $29(4)$ \\
\hline 9 & Acinetobacter baumannii (MDRO) & $1(0.2)$ & $2(1)$ & $3(0.4)$ \\
\hline 10 & Enterobacter species & $11(2)$ & $5(2.7)$ & $16(2.2)$ \\
\hline 11 & Proteus species & $6(1.1)$ & $4(2.2)$ & $10(1.4)$ \\
\hline 12 & C.koseri & $7(1.2)$ & $0(0 \%)$ & $7(0.9)$ \\
\hline 13 & C.freundii & $1(0.1)$ & $1(0.5)$ & $2(0.3)$ \\
\hline 14 & Morganella morgana & $0(0)$ & $2(1)$ & $2(0.3)$ \\
\hline \multirow[t]{2}{*}{15} & Serratia species & $1(0.1)$ & $0(0)$ & $1(0.1)$ \\
\hline & Total Gram Negative & $542(74)$ & $186(26)$ & $728(86)$ \\
\hline \multicolumn{5}{|c|}{ (b) Gram Positive cocci } \\
\hline 1 & Group "D" Streptococci & $41(47.1)$ & $23(74.2)$ & $64(54.2)$ \\
\hline 2 & Group “B” Streptococci & $31(35.6)$ & $6(19.3)$ & $37(31.4)$ \\
\hline 3 & S. aureus & $10(11.5)$ & $2(6.5)$ & $12(10.2)$ \\
\hline 4 & S. aureus (MRSA) & $4(4.6)$ & $0(0)$ & $4(3.4)$ \\
\hline \multirow[t]{3}{*}{5} & S. saprophyticus & $1(1.2)$ & $0(0)$ & $1(.8)$ \\
\hline & Total Gram Positive cocci & $87(74)$ & $31(26)$ & $118(14)$ \\
\hline & Total & $629(74)$ & $217(26)$ & $846(100)$ \\
\hline
\end{tabular}

Extended Spectrum Beta $(\beta)$ Lactamases (ESBLs), Multipple Drug Resistant Organism (MDRO),Carbapenem-Resistant Enterobacteriaceae (CRE).

Overall, gender wise variation was found in percentage prevalence of uropathogens among different age groups of UTI patients. The highest prevalence of uropathogens was found in age group II in females with the highest percentage of Gram positive than Gram negative .In age group II from males, Gram negative isolates was found more than females. The least uropathogens was observed in the patients of age group I in both males and females. Gram negative urinary isolates were prevalent the most in both genders as shown in Figure 1. 


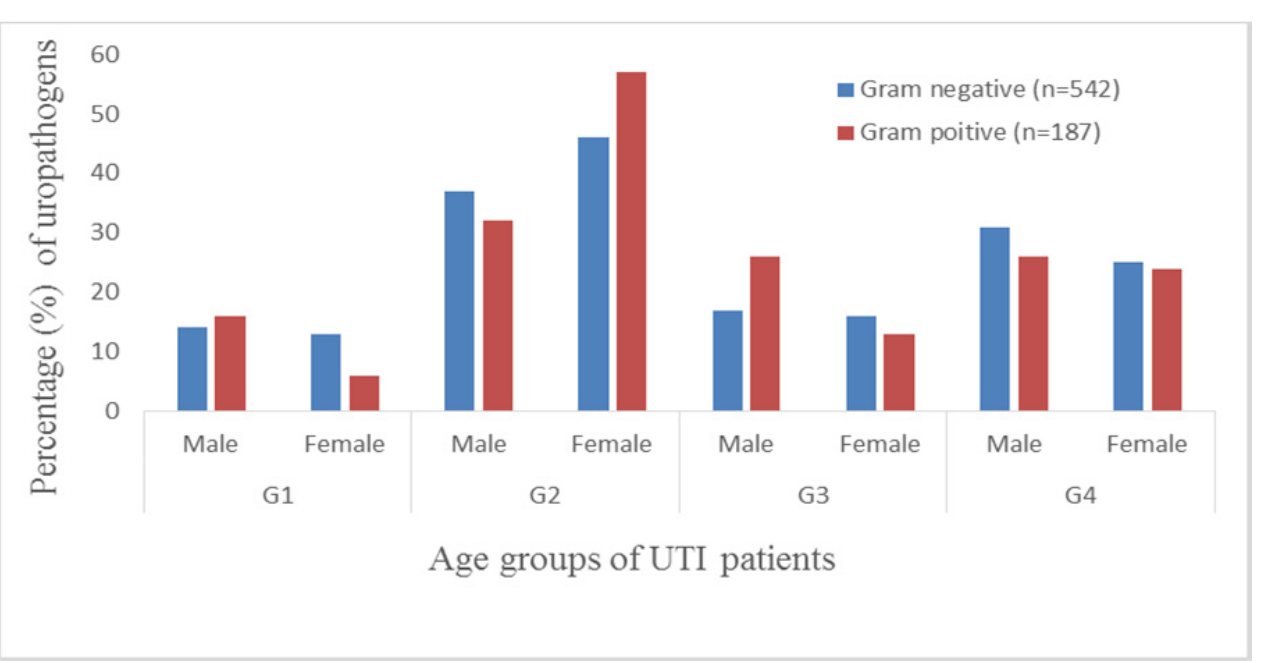

Patients of age $<12$ years were included in group I, 13-40 years in group II, 41-60 years in group III and $>60$ years are in group IV.

Figure 1. Age wise distribution of Gram (-/+) uropathogens among UTI patients

High variation was observed in antibiotic resistance pattern of uropathogens. The highest antibiotic resistance tendency (34.3\%) was noticed in E.coli against Nalidixic Acid, however the least resistant against Ceftriaxone, Ceftazidime, Ciprofloxacin and Nitrufurantoin among female and male genders. On the other hand $(24.8 \%)$ strains of E.coli were found positive for Extended Spectrum Beta Lactamase producers (ESBLs). E.coli (ESBLs) showed the highest (100\%) resistance to Amoxicillin/Clavulanic Acid, Ceftazidime and Ceftriaxone, similarly this group of uropathogens were least resistant to Amikacin, Colistin Sulphate, Meropenem, Polymyxin B and Nitrofurantoin. Klebsiella species were found the second frequently occurring Gram negative uropathogens, it has shown the highest (13.8\%) resistant to Nitrofurantoin and Nalidixic Acid, while the least resistant (1.3\%) to Ceftriaxone, Ceftazidime and Ciprofloxacin among female (Table 3) and male (Table 4). Besides this, (15\%) strains of Klebsiella species were positive for Extended Spectrum Beta $(\beta)$ Lactamase producers (ESBLs) .Similarly, Extended Spectrum Beta $(\beta)$ Lactamase producers (ESBLs) Klebsiella pneumoniae showed the highest (100\%) resistanace to Aoxicillin/Clavulanic Acid, Ceftazidime and Ceftriaxone like other Extended Spectrum Beta lactamase producers (ESBLs), however the least $(0 \%)$ resistant trend were observed against Amikacin, Colistin Sulphate, Meropenem and Polymyxin B among female and male genders. Pseudomonas species showed the maximum (5.7\%) resistant to Ceftazidime and Meropenem, while the least $(0 \%)$ resistant to Amikacin and Piperacillin/Tazobactam among female gender, however no resistance were observed against Amikacin, Ceftazidime, Ciprofloxacin, Meropenem and Piperacillin/Tazobactam among male gender. Moreover, (9.8\%) of Pseudomonas Species were Multi Drug Resistant Organism (MDRO) (Pseudomonas aeroginosa strains) that showed the highest resistant (100\%) to Ceftazidime, Ciprofloxacin, Meropenem and Piperacillin/Tazobatam among female gender, however these strains showed the least (0\%) resistance to Colistin Sulphate and Polymyxin $\mathrm{B}$ among both genders. Acinetobacter baumannii, exhibited the highest resistant to Ceftazidime and Ciprofloxacin among female and male genders, while this uropathogens were the least resistant $(0 \%)$ to Meropenem, Trimethoprim/Sulphamethoxazole and Piperacillin/Tazobactam among both genders. Similarly, $(9.4 \%)$ of $A$. baumannii strains were found Multi Drug Resistant Organisms (MDRO), these strains exhibited maximum (100\%) resistant to Amikacin, Ceftazidime, Ciprofloxacin, Meropenem, and Piperacillin/Tazobactam among female and male genders, however these strains showed the least resistant $(0 \%)$ to Colistin Sulphate and Polymyxin B in each gender. Enterobacter cloacae showed the highest (100\%) resistant pattern against Amoxicillin/Clavulanic acid, Nitrofurantoin and Cefuroxime among both genders, however this strain showed the least (18\%) resistant to Ceftazidime, Ciprofloxacin, and Nalidixic acid among female gender, however, $(0 \%)$ resistant were observed against Ceftazidime, Ciprofloxacin, and Nalidixic Acid among male gender. Citrobacter species, showed the peak $(100 \%)$ resistant pattern against Amoxicillin/Clavulanic acid among female gender, however these bacterium showed the highest (100\%) resistant to Aoxicillin/Clavulanic acid, Ciprofloxacin and Nalidixic acid among male gender, on the other hand, the least resistant were observed against Ciprofloxacin, Cefotaxime and Nitrofurantoin among female gender, however, these bacterium showed the least $(0 \%)$ resistant to Amikacin, Ceftazidime,Nitrofurantoin among male gender. Proteus species on the other hand, showed the highest resistant $(100 \%)$ pattern against Nitrofurantoin among both genders, however the least $(0 \%)$ resistant were observed against 
Ciprofloxacin, Ceftriaxone and Cefotaxime among female gender, however, the least $(0 \%)$ resistant were observed against Amoxicillin/Clavalonic acid, Ceftazidime, Ceftriaxone, Ciprofloxacin and Nalidixic Acid among male gender. Serratia species, the least encounter uropathogens isolated from female gender, showed the highest (100\%) resistant against Amoxicillin/Clavulanic acid, Cefuroxime, Nitrofurantoin and Nalidixic acid, however this uropathogens showed the least $(0 \%)$ resistant pattern against Ceftazidime, Ciprofloxacin and Cefotaxime. Table 4 showed the disparity in sensitivity pattern of uropathogens among males. K. pneumoniae $(0.8 \%) C R E$ (Carbapenem Resistant Enterobacteriacae) isolated from male gender only, showed the highest (100\%) resistance against Amikacin, Augmentin, Ceftazidime, Gentamicin, Ceftriaxone, Ciprofloxacin, Nitrofurantoin, Meropenem, Nalidixic Acid, however this strain showed no Resistant to Colistin Sulphate and Polymyxin. Similarly, Morganella morganii were isolated from male gender only, showed the highest $(100 \%)$ resistant pattern against Amoxicillin/Clavalonic acid, Cefuroxime and Nitrofurantoin, however this bacterium show the least $(0 \%)$ resistant to Ceftazidime and Ciprofloxacin.

Table 3. Percentages (\%) of antimicrobial resistance pattern among Gram negative isolates from females

\begin{tabular}{|c|c|c|c|c|c|c|c|c|c|c|c|c|c|c|c|c|c|c|c|}
\hline & $\begin{array}{l}1 \\
\vdots \\
\vdots\end{array}$ & $\underset{5}{\mathbb{E}}$ & i & 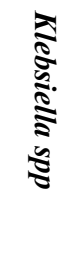 & 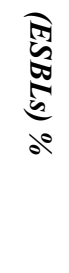 & 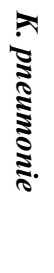 & 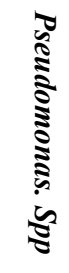 & 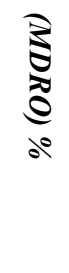 & 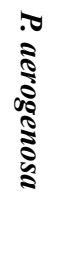 & 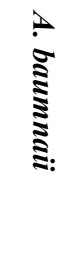 & 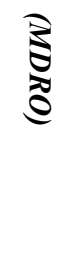 & 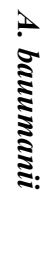 & 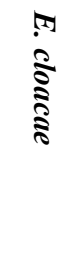 & $\begin{array}{l}\Omega \\
\text { है } \\
\vdots \\
\vdots\end{array}$ & 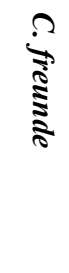 & $\xi$ & 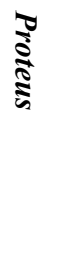 & $\grave{s}$ & 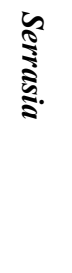 \\
\hline AK30 & NT & 0 & & NT & 0 & & 0 & 0 & & NT & 100 & & NT & NT & 0 & NT & & NT & \\
\hline $\begin{array}{l}\text { AMC } \\
120 / 10\end{array}$ & 10.4 & 100 & & 8.3 & 100 & & NT & NT & & NT & NT & & 100 & 100 & 100 & 16.6 & & 100 & \\
\hline CAZ30 & 4.7 & 100 & & 4.1 & 100 & & 5.7 & 100 & & 26.0 & 100 & & 18.1 & 0 & 100 & NT & & 0 & \\
\hline CN10 & NT & NT & & NT & NT & & 2.8 & 50 & & NT & NT & & NT & NT & NT & NT & & NT & \\
\hline CRO30 & 3.3 & 100 & & 1.3 & 100 & & NT & NT & & NT & NT & & NT & NT & NT & 0 & & NT & \\
\hline $\begin{array}{l}\text { CXM3 } \\
0\end{array}$ & NT & NT & & NT & NT & & NT & NT & & NT & NT & & 100 & NT & NT & NT & & 100 & \\
\hline CIP5 & 5.0 & 56.9 & & 5.5 & 28.5 & & 2.8 & 100 & & 21.7 & 100 & & 18.1 & 0 & 0 & 0 & & 0 & \\
\hline CT10 & NT & 0 & & NT & 0 & & NT & 0 & & NT & 0 & & NT & NT & NT & NT & & NT & \\
\hline CTX30 & 3.0 & NT & & NT & NT & & NT & NT & & 30.4 & 100 & & 18.1 & 0 & 0 & 0 & & 0 & \\
\hline F300 & 0.6 & 2.5 & & 13.8 & 14.2 & & NT & NT & & NT & NT & & 100 & 0 & 0 & 100 & & 100 & \\
\hline $\begin{array}{l}\text { MEM1 } \\
\text { 0 }\end{array}$ & NT & 0 & & NT & 0 & & 5.7 & 100 & & 0 & 100 & & NT & NT & 0 & NT & & NT & \\
\hline NA30 & 34.3 & 55.6 & & 13.8 & 14.2 & & NT & NT & & NT & NT & & 18.1 & 0 & 100) & 16.6 & & 100 & \\
\hline PB300 & NT & 0 & & NT & 0 & & NT & 0 & & NT & 0 & & NT & NT & 0 & NT & & NT & \\
\hline SXT25 & $\mathrm{NT}$ & 45.5 & & NT & 42.8 & & NT & NT & & 0 & 100 & & NT & NT & 0 & NT & & NT & \\
\hline $\begin{array}{l}\text { TZP } \\
100 / 10\end{array}$ & NT & NT & & NT & NT & & 0 & 100 & & 0 & 100 & & NT & NT & NT & NT & & NT & \\
\hline
\end{tabular}

AK, Amikacin, AMC, Augmentin, CAZ, Ceftazidime, CN, Gentamicin, CRO, Ceftriaxone, CXM , Cefuroxime, CIP, Ciprofloxacin, CT, Colistin Sulphate, CTX, Cefotaxime, F, Nitrofurantoin, MEM, Meropenem, NA, Nalidixic Acid, PB, Polymxin B, SXT ,Trimethoprim/Sulphamethoxazole, TZP, PiperacillinTazobactam, NT, Not Tested. 
Table 4. Percentages (\%) of antimicrobial resistance pattern among Gram negative isolates from males

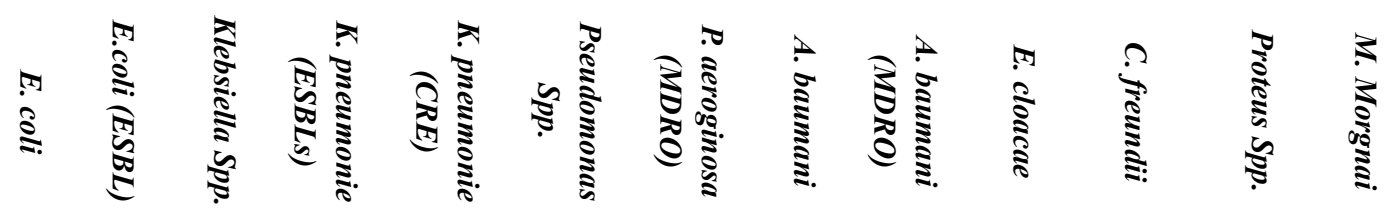

\begin{tabular}{llllllllllllll}
\hline *AK30 & NT & 0 & NT & 0 & 100 & 0 & 100 & NT & NT & NT & 0 & NT & NT \\
AMC20/10 & 4.3 & 100 & 3.4 & 100 & 100 & NT & NT & NT & NT & 100 & 100 & 0 & 100 \\
CAZ30 & 0 & 100 & 0 & 100 & 100 & 0 & 66.6 & 16.6 & 100 & 0 & 0 & 0 & 0 \\
CN10 & NT & NT & NT & NT & 100 & 0 & 100 & NT & NT & NT & NT & NT & NT \\
CRO30 & 0 & 100 & 0 & 100 & 100 & NT & NT & NT & NT & NT & NT & 0 & NT \\
CXM30 & NT & NT & NT & NT & NT & NT & NT & NT & NT & 100 & NT & NT & 100 \\
CIP5 & 7.2 & 61.9 & 0 & 45.4 & 100 & 0 & 100 & 16.6 & 100 & 0 & 100 & 0 & 0 \\
CT10 & NT & 0 & NT & 0 & 0 & NT & 0 & NT & 0 & NT & NT & NT & NT \\
CTX30 & NT & NT & NT & NT & NT & NT & NT & NT & NT & NT & NT & NT & NT \\
F300 & 1.4 & 0 & 10.3 & 18.1 & 100 & NT & NT & NT & NT & 100 & 0 & 100 & 100 \\
MEM10 & NT & 0 & NT & 0 & 100 & 0 & 66.6 & 0 & 100 & NT & NT & NT & NT \\
NA30 & 39.1 & 85.7 & 6.8 & 27.2 & 100 & NT & NT & NT & NT & 0 & 100 & 0 & 50 \\
PB300 & NT & 0 & NT & 0 & 0 & NT & 0 & NT & 0 & NT & NT & NT & NT \\
SXT25 & NT & 21.4 & NT & 72.7 & NT & NT & NT & 0 & 50 & NT & 100 & NT & NT \\
TZP100/10 & NT & NT & NT & NT & NT & 0 & 100 & 0 & 100 & NT & NT & NT & NT \\
& & & & & & & & & & & & &
\end{tabular}

AK, Amikacin, AMC, Augmentin, CAZ, Ceftazidime, CN, Gentamicin, CRO, Ceftriaxone, CXM, Cefuroxime, CIP, Ciprofloxacin, CT, Colistin Sulphate, CTX, Cefotaxime ,F, Nitrofurantoin, MEM, Meropenem, NA, Nalidixic Acid, PB, Polymxin B, SXT, Trimethoprim/Sulphamethoxazole, TZP, Piperacillin/Tazobactam. ESBLs, Extended Spectrum Beta Lactamases, CRE, Carbapenem Resistant Enterobacteriaceae, NT, Not Tested.

Among the Gram-positive isolates, S. aureus (MRSA) isolated from female gender only, exhibited utmost resistance (100\%) against Amoxacillin/Clavalonic acid, Cefotaxime, Cefoxitin and Oxacillin, and least $(0 \%)$ Resistant was observed against Ciprofloxacin, Nitrofurantoin, Rifampicin, linezolid, Vancomycin among female gender, have shown in Table 5. 
Table 5. Percentages (\%) of antimicrobial resistance pattern among Gram $(+)$ isolates from females

\begin{tabular}{|c|c|c|c|c|c|c|c|c|c|c|c|c|c|c|c|c|c|}
\hline & $\underset{\ominus}{\stackrel{2}{e}}$ & 常 & $\underset{\gamma}{2}$ & 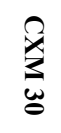 & $\begin{array}{l}\mathcal{3} \\
\tilde{0} \\
\tilde{\theta}\end{array}$ & $\begin{array}{l}2 \\
\vec{x} \\
\stackrel{\omega}{0}\end{array}$ & 氙 & $\stackrel{\vec{v}}{\vec{v}}$ & 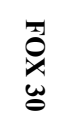 & $\underset{\mathfrak{v}}{\tilde{y}}$ & $\underset{v}{Z}$ & $\underset{v}{\pi}$ & $\stackrel{0}{x}$ & Ð & 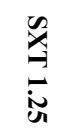 & 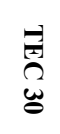 & $\underset{⿱ 乛}{\infty}$ \\
\hline GP"D" & 0 & 7.3 & 14.6 & NT & NT & NT & 0 & NT & NT & NT & NT & NT & NT & NT & NT & 0 & 41 \\
\hline \multicolumn{18}{|l|}{ Streptococci } \\
\hline GP“B” & 0 & 0 & 0 & 0 & 0 & NT & 0 & NT & NT & NT & NT & NT & NT & 0 & NT & NT & NT \\
\hline \multicolumn{18}{|l|}{ Streptococci } \\
\hline S. aureus & 0 & NT & 10 & NT & NT & 0 & 0 & 10 & 0 & NT & NT & NT & 0 & NT & 0 & NT & NT \\
\hline MRSA & 100 & NT & 0 & NT & NT & 100 & 0 & 25 & 100 & 0 & NT & 0 & 100 & NT & 25 & NT & 0 \\
\hline$S$ & 0 & NT & 0 & NT & 0 & NT & 0 & NT & NT & NT & 100 & NT & NT & NT & 0 & NT & NT \\
\hline saprophytics & & & & & & & & & & & & & & & & & \\
\hline
\end{tabular}

AMC, Augmentin, AMP, Ampicillin, CIP, Ciprofloxacin, CXM, Cefuroxime, CRO, Ceftriaxone, CTX, Cefotaxime, E, Erythromycin, F, Nitrofurantoin, , SXT, Trimethoprim/Sulphamethoxazole,FOX, Cefoxitin, LZ, Linzolid, NOV, Novobiocin, RD, Rifampacin, OX, Oxacillin, P, Penicillin, TE, Techoplanin, VA, Vancomycin, NT, Not Tested, GP, Group, MRSA, Methicillin Resistance Staph Aureus.

Group "B" Streptococci were found high sensitive to Augmentin, Ampicillin, Ciprofloxacin, Ceftriaxone and Cefuroxime among both genders however, no resistant were observed against Augmentin, Ampicillin, Ciprofloxacin, Ceftriaxone and Cefuroxime. S. saprophytics showed the highest (100\%) Resistant to Novobiocin and the least $(0 \%)$ to Amoxicillin/Clavalonic acid, Ciprofloxacin, Nitrofurantoin, Ceftriaxone and Trimethoprim/Sulphamethaxazole. Group"D" Streptococci (Enterococcus faeculis) presented high resistance (14.6\%) to Ciprofloxacin and Ampicillin (7.3\%) among female gender and Ciprofloxacin (34.7\%), Ampicillin $(13 \%)$ and Nitrofurantoin $(8.6 \%)$ among male gender, this bacterium showed the least $(0 \%)$ Resistant to Amoxacillin/Clavalonic acid and Vancomycin among female and male genders respectively as shown in Table 6 .

Table 6. Percentages (\%) of antimicrobial resistance pattern among Gram $(+)$ isolates from males

\begin{tabular}{|c|c|c|c|c|c|c|c|c|c|c|c|c|c|c|}
\hline & AMC & AMP & CIP & CXM & CRO & CTX & $F$ & E15 & FOX & OX1 & P10 & SXT & TEC & VA \\
\hline & $120 / 10$ & 10 & 5 & 30 & 30 & 30 & 300 & & 30 & & & 1.25 & 30 & 30 \\
\hline Gp "D" & 0 & 13 & 34.7 & NT & NT & NT & 8.6 & NT & NT & NT & NT & NT & 0 & 0 \\
\hline Streptococci & & & & & & & & & & & & & & \\
\hline $\begin{array}{l}\text { Gp "B" } \\
\text { Streptococci }\end{array}$ & 0 & 0 & 0 & 0 & 0 & NT & 0 & NT & NT & NT & 0 & NT & NT & NT \\
\hline S.aureus & 0 & NT & 0 & NT & NT & 0 & NT & 0 & 0 & 0 & NT & 0 & NT & 0 \\
\hline
\end{tabular}

AMC, Augmentin, AMP, Ampicillin, CIP, Ciprofloxacin, CXM, Cefuroxime, CRO, Ceftriaxone, CTX, Cefotaxime, E, Erythromycin, F, Nitrofurantoin, SXT, Trimethoprim/Sulphamethoxazole, FOX, Cefoxitin, LZ, Linzolid, NOV, Novobiocin, RD, Rifampacin, OX, Oxacillin, P, Penicillin, TE, Techoplanin, VA, Vancomycin, NT, Not Tested, GP, Group, MRSA, Methicillin Resistance Staph Aureus

\section{Discussion}

The purpose of current study to focus the condition of antimicrobial resistant pattern in uropathogens to monitor and support to improve treatment of urinary tract infections (UTI). It is a retrospective study where routine diagnostic results and susceptibility analysis are exercise and used. The results may not reflect the true prevalence as most patients are treated empirically for Urinary Tract Infection (UTIs). This study indicated that UTI is more common in female (74\%) than males $(26 \%)$ that is similar with previous study in 2017 , India with $73.97 \%$ in females and 26.02\%) UTI prevalence to males (Lawhale \& Naikwade, 2017) and other studies (Dash, Padhi, 
Mohanty, Panda, \& Parida, 2013; Oladeinde, Omoregie, Olley, \& Anunibe, 2011; Shah, Wasim, \& Abdullah, 2015). Possible reasons for common UTI in females including short urethra, close proximity of vagina with urethral meatus, rich microbial flora with rectal mucosa and sexual intercourse have been reported (Oladeinde et al., 2011; Shah et al., 2015). Current study observed high UTI in females of age group 13-40 years. This age group reported high risk for UTI cases and contribute highly sexually active women group (Dash et al., 2013; Nalini, Meenakshi, \& Ramya, 2013; Shaifali, Gupta, Mahmood, \& Ahmed, 2012). Nalini et al. (2013) and Dash et al. (2013) also presented the same results. Among males, the most common UTI influenced age group was 13-40 years unlike other study that reported $44.4 \%$ age group $61-80$ years (Lawhale \& Naikwade, 2017).

Among total 4,480 urine samples, $846(18.8 \%)$ sample were positive for significant bacteriuria. Gram-negative organisms however, were the most frequent uropathogens, which account for $(86 \%)$ UTI cases. This study results were found similar to the research conducted in Sultan Qaboos Uiniversity hospital, Oman, reported in a literature (El-Naggari et al., n.d.). Results from present study have shown E.coli the most common prevalent (66.9\%) uropathogens, in comparison with other studies from Pakistan that indicated the E. coli prevalence (62\%) to (68\%) in urinary samples (Keah, Wee, Chng, \& Keah, 2007; Kothari \& Sagar, 2008). Klebsiella species was the second highest prevalent uropathogens accounting for (16.5\%) of cases. Studies from United Kingdom (UK) reported 8-26\% occurrence of Klebsiella species in UTI cases (Hasan et al., 2007). Our study results revealed the highest (16.6\%) presence of ESBL producing E. coli and followed by (16.5\%) Klebsiella species, (15\%) Klebsiella pneumonia ESBLs prducers, Pseudomonas species (7\%), A. baumannii (4.4\%), Enterobacter species (2.2\%), Proteus species (1.4\%), Citrobacter species (1.2\%), M.morgani (0.3\%) and Serratia species (0.1\%) respectively.

Results from this study exhibited that (14\%) UTI were contributed through Gram-positive cocci. Group "D" Streptococci was the most frequent (54.2\%) uropathogens and the prevalence of these organisms were found higher in male than female $(74.2 \%)$ and $(47.1 \%)$ respectively. Whereas group "B" Streptococci were the second frequently $(31.4 \%)$ occurring Gram positive uropathogens, though the occurrence of this organism was higher in female than male (35.6\%) and (19.3\%) respectively. A previous study indicated the prevalence of Gram positive uropathogens including E. faecalis (15\%) followed by S. aureus (1\%) (Sohail, Khurshid, Saleem, Javed, \& Khan, 2015).

The results indicated the variations in sensitivity pattern of gram negative uropathogens among males and female. Nalidixic acid and Ciprofloxacin showed the highest $34.3 \%$ and $10 \%$ resistant pattern to E. coli. In comparison with other study conducted in India that found E. coli more high resistance to antibiotics like doxycycline (65\%), cephalosporins (60\%) and levofloxacin (52.01\%) (Lawhale \& Naikwade, 2017). Nitrofurantoin and Cefotaxime followed by Ceftriaxone and Ceftazidime were found susceptible in females against males. Whereas in males sensitivity were observed against Ceftazidime, Ceftriaxone and Nitrofurantoin.

ESBLs producing E. coli have exhibited the peak resistant (100\%) to Penicillin, Cephalosporin and Azetrenom (Augmentin, Ceftazidime and Ceftriaxone) for both genders. Similar findings were presented in other studies with $100 \%, 100 \%$ resistant to ampicillin and $92 \%, 80.8 \%$ resistant to non ESBL producing E.coli respectively (Elsayed, Ismail, \& Elgamal, 2017; Islam et al., 2015). However, ESBLs producing E. coli displayed the sensitivity to Amikacin, Polymyxin B, Colistin Sulphate and Meropenem. Klebsiella species presented the highest resistance to Nalidixic Acid and Nitrofurantoin among female and male genders. In previous studies from India, K. pneumoniae showed resistance to doxycycline (82.85\%) cephalosporin (63.33\%), levofloxacin 75.23\% (Dash et al., 2013; Fajfr et al., 2017; Sood, Malhotra, Das, \& Kapil, 2008). These species revealed the susceptibility to Ceftriaxone and Ceftazidime followed by Ciprofloxacin and Amoxicillin/Clavalonic acid among female, in comparison to male, Klebsiella species showed sensitivity against Ceftriaxone, Ceftazidime, and Ciprofloxacin, followed by Amoxicillin/Clavalonic. Penicillin, Cephalosporin and Azetrenom group (e.g. Augmentin, Ceftazidime and Ceftriaxone) were found resistant to ESBL producing K. pneumoniae among both male and female genders. Likewise, these species exhibited susceptibility to Amikacin, Colistin Sulphate, Polymyxin B and Meropenem.

Pseudomonas species showed the highest resistance from female to Ceftazidime and Meropenem followed by Ciprofloxacin and Gentamycin, however these species were observed sensitive against Amikacin and Piperacillin /Tazobactam. The resistance pattern among male were dissimilar as no resistant was observed against antibiotic like Amikacin, Ceftazidime, Gentamycin, Cirofloxacin, Meropenem and Piperacillin/Tazobactam. In contrary, P.aeroginosa (MDRO) showed the highest resistance to Ceftazidime, Ciprofloxacin and Meropenem but showed the susceptibility to Amikacin, Polymyxin B and Colistin Sulphate among female, however, the resistance profile showed a different representation in male, with the highest resistance pattern to Amikacin, Ciprofloxacin, Gentamycin and Piperacillin/Tazobactam. Similarly, the sensitivity was observed in the same against Colistin Sulphate, Polymyxin B, followed by Ceftazidime and Meropenem. A.baumannii exhibited the highest resistance 
among female to Cefotaxime and Ceftazidime followed by Ciprofloxacin, and sensitivity to Meropenem, Trimethoprim/Sulphamethoxazole and Piperacillin/Tazobactam.

A study also reported the increasing resistance against ampicillin, Cephlothin and Trimethoprim/Sulphamethoxazole (K. Gupta, Hooton, \& Stamm, 2001). However, an altered resistance pattern was detected among male by showing the highest resistant to Ceftazidime and Ciprofloxacin, while the sensitivity were observed against Meropenem, Trimethoprim/Sulphamethxazole and Piperacillin/Tazobactam. In contrast, some MDRO strains of A.baumannii were isolated too, which showed the highest resistance to Amikacin, Ceftazidime, Cefotaxime, Ciprofloxacin, Trimethoprim/sulphamethoxazole, Piperacillin/Tazobactam and Meropenem among female and male genders. This group of microorganisms displayed the minimum resistance to Polymyxin B and colistin Sulphate in both genders. E. cloacae comparatively showed the highest resistance to Augmentin, Nitrofurantoin and Cefuroxime in both genders; that showed sensitivity against Ceftazidime, Ciprofloxacin, Cefotaxime and Nalidixixc Acid among female and male genders. C. koseri isolated in female only, which showed the highest resistance to Augmentin, however they showed the least resistance to Ceftazidime, Ciprofloxacin, Cefotaxime, Nitrofurantoin and Nalidixic Acid. On the hand, C. freundii showed the highest resistant to Augmentin, Ceftazidime and Nalidixic Acid, while susceptibility against Amikacin, Ciprofloxacin, Cefotaxime, Nitrofurantoin, Meropenem and Trimethoprim/Sulphamethaxazole among females. However, among male C. freundii revealed different resistant pattern, the highest resistant to Augmentin, Ciprofloxacin, Nalidixic Acid and Trimethoprim/Sulphamethaxazole while sensitivity to Amikacin, Ceftazidime and Nitrofurantoin.

Proteus species were found resistant to Nitrofurantoin, Augmentin and Nalidixic Acid, however susceptibility profile were observed against Ceftriaxone and Ciprofloxacin. In comparison with other study that showed Proteus resistance against 12 different antibiotis but sensitive to piperacillin/tazobactam, meropenem and cefoperazone/sulbactam (Sohail et al., 2015).But unlike females a different resistant pattern were observed in males, showing the peak resistant to Nitrofurantoin, however sensitivity were observed against Augmentin, Ceftazidime, Ceftriaxone, Ciprofloxacin and Nalidixic Acid. Among female, Serratia species were found less frequent uropathogens, which have shown the highest resistant against Augmentin, Cefuroxime, Nitrofurantoin and Nalidixic Acid. However, the sensitivity was observed against Ceftazidime, Ciprofloxacin and Cefotaxime. Likewise, less prevalent uropathogens among male were M.morganaii showed the highest resistant pattern to Augmentin, Cefuroxime, Nitrofurantoin and Nalidixic Acid, nevertheless, susceptibility were observed against Ceftazidime and Ciprofloxacin. A study reported that all Gram negative uropathogens have sensitivity against Fosphomycin that indicated the first choice of drug for UTI treatment. ESBL producing and MDR microorganisms also had sensitivity against Nitrofurantoin and Fosfomycin that also can be used for UTI management (Das et al., 2006; V. Gupta, Rani, Singla, Kaistha, \& Chander, 2013; Nalini et al., 2013).

Among the Gram-positive isolates S. aureus (MRSA) exhibited utmost resistance (100\%) against Augmentin, Cefotaxime, Cefoxitin and Oxacillin and high sensitivity was observed among females about Ciprofloxacin, Nitrofurantoin, linezolid, and vancomycin. Group“B” Streptococci was found high sensitive to Augmentin, Ampicillin, Ciprofloxacin, and Cefuroxime. S. saprophytics presented high resistance (100\%) to Novobiocin. Group "D" Streptococci showed the least resistant to Amoxicillin/Clavalonic acid, Vancomycin, Techoplanin among female and male genders.In previous study Amikacin, Vancomycin, linezolid, Fosfomycin, fusidic acid, and clindamycin had strong antibacterial activity against the Gram-positive uropathogens (Sohail et al., 2015). Group "D" Streptococci among males indicated the highest resistant (34.7\%,) (13\%) and (8.6\%) against Ciprofloxacin, Nitrofurantoin, and Ampicillin respectively This high antibiotics resistance might be due to self-medication in poor countries, extensive use of broad spectrum antibiotics (Dash et al., 2013).

\section{Conclusion}

E.coli is the most prevalent organism in urinary tract infection (UTI) in this study. $\beta$-lactam antibiotics, Cephalosporins, Fluroquiolones, Macrobids would be the first line of drugs and most effective for the empirical treatment of Gram-negative and Gram-positive uropathogens respectively; however, Aminoglycosides, Carbapenems and Polymyxins could be used for the treatment of UTI infections as the second choice. Furthermore, this study highlight the increased prevalence of antibiotic Resistance to ESBL strains, MDRO which is a challenge to the medical field. The usage of antibacterial drugs should be monitor to avoid the day by day increasing resistance and for the proper management of UT Infections.

\section{Study Limitation}

Generalization of result cannot be done as the study was limited to only one region of Oman. Further, studies in other regions need to be conducted in order to find out the impact of resistance to clinical management and patient outcome. 


\section{Authors Contribution}

MK composed the manuscript, MKA perform interpretation of results, HGM did the final revision and critical review, MMU and AYMDAB planed the study and accomplished the statistical analysis.

\section{Acknowledgements}

The authors are thankful to hospital administration and laboratory team for their support during entire study period.

\section{Competing Interests Statement}

There is no conflict of interest among authors.

\section{References}

Barrow, G., \& Feltham, R. (1993). Cowan and Steel's Manual for the Identification of Medical Bacteria. Cambridge, Press Syndicate of the University of Cambridge. https://doi.org/10.1017/CBO9780511527104

Boyle, V. J., Fancher, M. E., \& Ross, R. W. (1973). Rapid, Modified Kirby-Bauer Susceptibility Test with Single, High-Concentration Antimicrobial Disks. Antimicrobial agents and chemotherapy, 3(3), 418-424. https://doi.org/10.1128/AAC.3.3.418

Chen, Y.-H., Ko, W.-C., \& Hsueh, P.-R. (2013). Emerging Resistance Problems and Future Perspectives in Pharmacotherapy for Complicated Urinary Tract Infections. Expert opinion on pharmacotherapy, 14(5), 587-596. https://doi.org/10.1517/14656566.2013.778827 PMid:23480061

Courvalin, P. (2006). Vancomycin Resistance in Gram-Positive Cocci. Clinical Infectious Diseases, 42(Supplement_1), S25-S34. https://doi.org/10.1086/491711

Das, R., Chandrashekhar, T., Joshi, H., Gurung, M., Shrestha, N., \& Shivananda, P. (2006). Frequency and Susceptibility Profile of Pathogens Causing Urinary Tract Infections at a Tertiary Care Hospital in Western Nepal. Singapore medical journal, 47(4), 281.

Dash, M., Padhi, S., Mohanty, I., Panda, P., \& Parida, B. (2013). Antimicrobial Resistance in Pathogens Causing Urinary Tract Infections in a Rural Community of Odisha, India. Journal of family \& community medicine, 20(1), 20. https://doi.org/10.4103/2230-8229.108180

Desai, D. J., Gilbert, B., \& McBride, C. A. (2016). Paediatric Urinary Tract Infections: Diagnosis and Treatment. Australian family physician, 45(8), 558. PMid:27610444

El-Naggari, M., Waadallah, S., Al Nabhani, D., Al Sawai, A., Al Muharrmi, Z., \& El Nour, I. (n.d.). Incidence of Antibiotics Resistance of Uropathogens in Omani Children Presented with Single Episode of Urinary Tract Infection.

Elsayed, T., Ismail, H., \& Elgamal, S. (2017). The Occurrence of Multidrug Resistant E. Coli Which Produce Esbl and Cause Urinary Tract Infections. J Appl Microbiol Biochem, 2, 1.

Fajfr, M., Louda, M., Paterová, P., Ryšková, L., Pacovský, J., Košina, J., . . Brod’ák, M. (2017). The Susceptibility to Fosfomycin of Gram-Negative Bacteria Isolates from Urinary Tract Infection in the Czech Republic: Data from a Unicentric Study. BMC urology, 17(1), 33. https://doi.org/10.1186/s12894-017-0222-6

Fisher, J. F., Kavanagh, K., Sobel, J. D., Kauffman, C. A., \& Newman, C. A. (2011). Candida Urinary Tract Infection: Pathogenesis. Clinical Infectious Diseases, 52(suppl_6), S437-S451. https://doi.org/10.1093/cid/cir110

Foxman, B. (2010). The Epidemiology of Urinary Tract Infection. Nature Reviews Urology, 7(12), 653-660. https://doi.org/10.1038/nrurol.2010.190

Foxman, B. (2014). Urinary Tract Infection Syndromes: Occurrence, Recurrence, Bacteriology, Risk Factors, and Disease Burden. Infectious disease clinics of North America, 28(1), 1-13. https://doi.org/10.1016/j.idc.2013.09.003

Garau, J. (2008). Other Antimicrobials of Interest in the Era of Extended - Spectrum B - Lactamases: Fosfomycin, Nitrofurantoin and Tigecycline. Clinical Microbiology and Infection, 14(s1), 198-202. https://doi.org/10.1111/j.1469-0691.2007.01852.x

Gupta, K., \& Bhadelia, N. (2014). Management of Urinary Tract Infections from Multidrug-Resistant Organisms. Infectious Disease Clinics, 28(1), 49-59. https://doi.org/10.1016/j.idc.2013.10.002

Gupta, K., Hooton, T. M., \& Stamm, W. E. (2001). Increasing Antimicrobial Resistance and the Management of Uncomplicated Community-Acquired Urinary Tract Infections. Annals of internal medicine, 135(1), 41-50. 
https://doi.org/10.7326/0003-4819-135-1-200107030-00012

Gupta, V., Rani, H., Singla, N., Kaistha, N., \& Chander, J. (2013). Determination of Extended-Spectrum B-Lactamases and Ampc Production in Uropathogenic Isolates of Escherichia Coli and Susceptibility to Fosfomycin. Journal of laboratory physicians, 5(2), 90. https://doi.org/10.4103/0974-2727.119849

Hanna-Wakim, R. H., Ghanem, S. T., El Helou, M. W., Khafaja, S. A., Shaker, R. A., Hassan, S. A., . . Hajar, F. M. (2015). Epidemiology and Characteristics of Urinary Tract Infections in Children and Adolescents. Frontiers in cellular and infection microbiology, 5 . https://doi.org/10.3389/fcimb.2015.00045

Hannan, T. J., Totsika, M., Mansfield, K. J., Moore, K. H., Schembri, M. A., \& Hultgren, S. J. (2012). HostPathogen Checkpoints and Population Bottlenecks in Persistent and Intracellular Uropathogenic Escherichia Coli Bladder Infection. FEMS microbiology reviews, 36(3), 616-648. https://doi.org/10.1111/j.1574-6976.2012.00339.x

Hasan, A. S., Nair, D., Kaur, J., Baweja, G., Deb, M., \& Aggarwal, P. (2007). Resistance Patterns of Urinary Isolates in a Tertiary Indian Hospital. J Ayub Med Coll Abbottabad, 19(1), 39-41.

Hooton, T. M. (2012). Uncomplicated Urinary Tract Infection. New England Journal of Medicine, 366(11), 1028-1037. https://doi.org/10.1056/NEJMcp1104429

Islam, M. S., Yusuf, M. A., Begum, S. A., Sattar, A. A., Hossain, A., \& Roy, S. (2015). Extended-Spectrum-Beta-Lactamase Producing Uropathogenic Escherichia Coli Infection in Dhaka, Bangladesh. African Journal of Bacteriology Research, 7(1), 1-7.

Jacobsen, S., Stickler, D., Mobley, H., \& Shirtliff, M. (2008). Complicated Catheter-Associated Urinary Tract Infections Due to Escherichia Coli and Proteus Mirabilis. Clinical microbiology reviews, 21(1), 26-59. https://doi.org/10.1128/CMR.00019-07

Kass, E. H. (2002). Asymptomatic Infections of the Urinary Tract. The Journal of urology, 167(2), 1016-1020. https://doi.org/10.1016/S0022-5347(02)80328-7

Keah, S. H., Wee, E. C., Chng, K. S., \& Keah, K. C. (2007). Antimicrobial Susceptibility of Community-Acquired Uropathogens in General Practice. Malaysian family physician: the official journal of the Academy of Family Physicians of Malaysia, 2(2), 64.

Kline, K. A., Schwartz, D. J., Lewis, W. G., Hultgren, S. J., \& Lewis, A. L. (2011). Immune Activation and Suppression by Group B Streptococcus in a Murine Model of Urinary Tract Infection. Infection and immunity, 79(9), 3588-3595. https://doi.org/10.1128/IAI.00122-11

Kostakioti, M., Hultgren, S. J., \& Hadjifrangiskou, M. (2012). Molecular Blueprint of Uropathogenic Escherichia Coli Virulence Provides Clues toward the Development of Anti-Virulence Therapeutics. Virulence, 3(7), 592-593. https://doi.org/10.4161/viru.22364

Kothari, A., \& Sagar, V. (2008). Antibiotic Resistance in Pathogens Causing Community-Acquired Urinary Tract Infections in India: A Multicenter Study. The Journal of Infection in Developing Countries, 2(05), 354-358. https://doi.org/10.3855/jidc.196

Lawhale, M. A., \& Naikwade, R. (2017). Recent Pattern of Drug Sensitivity of Most Commonly Isolated Uropathogens from Central India. International Journal of Research in Medical Sciences, 5(8), 3631-3636. https://doi.org/10.18203/2320-6012.ijrms20173576

Levison, M. E., \& Kaye, D. (2013). Treatment of Complicated Urinary Tract Infections with an Emphasis on Drug-Resistant Gram-Negative Uropathogens. Current infectious disease reports, 15(2), 109-115. https://doi.org/10.1007/s11908-013-0315-7

Nalini, R., Meenakshi, B., \& Ramya, J. E. (2013). Recent Sensitivity Pattern of Escherichia Coli in Urinary Tract Infection. Paper presented at the INDIAN JOURNAL OF PHARMACOLOGY.

Nielubowicz, G. R., \& Mobley, H. L. (2010). Host-Pathogen Interactions in Urinary Tract Infection. Nature Reviews Urology, 7(8), 430-441. https://doi.org/10.1038/nrurol.2010.101

Oladeinde, B. H., Omoregie, R., Olley, M., \& Anunibe, J. A. (2011). Urinary Tract Infection in a Rural Community of Nigeria. North American journal of medical sciences, 3(2), 75. https://doi.org/10.4297/najms.2011.375

Ong, D. S., Kuyvenhoven, M. M., Van Dijk, L., \& Verheij, T. J. (2008). Antibiotics for Respiratory, Ear and Urinary Tract Disorders and Consistency among Gps. Journal of Antimicrobial Chemotherapy, 62(3), 587-592. https://doi.org/10.1093/jac/dkn230 
Orasch, C., Marchetti, O., Garbino, J., Schrenzel, J., Zimmerli, S., Mühlethaler, K., . . . Zbinden, R. (2014). Candida Species Distribution and Antifungal Susceptibility Testing According to European Committee on Antimicrobial Susceptibility Testing and New Vs. Old Clinical and Laboratory Standards Institute Clinical Breakpoints: A 6 - Year Prospective Candidaemia Survey from the Fungal Infection Network of Switzerland. Clinical microbiology and infection, 20(7), 698-705. https://doi.org/10.1111/1469-0691.12440

Paterson, D. L. (2006). Resistance in Gram-Negative Bacteria: Enterobacteriaceae. The American journal of medicine, 119(6), S20-S28. https://doi.org/10.1016/j.amjmed.2006.03.013

Pendleton, J. N., Gorman, S. P., \& Gilmore, B. F. (2013). Clinical Relevance of the Eskape Pathogens. Expert review of anti-infective therapy, 11(3), 297-308. https://doi.org/10.1586/eri.13.12

Ronald, A. (2002). The Etiology of Urinary Tract Infection: Traditional and Emerging Pathogens. The American journal of medicine, 113(1), 14-19. https://doi.org/10.1016/S0002-9343(02)01055-0

Shah, D. A., Wasim, S., \& Abdullah, F. E. (2015). Antibiotic Resistance Pattern of Pseudomonas Aeruginosa Isolated from Urine Samples of Urinary Tract Infections Patients in Karachi, Pakistan. Pakistan journal of medical sciences, 31(2), 341.

Shaifali, I., Gupta, U., Mahmood, S. E., \& Ahmed, J. (2012). Antibiotic Susceptibility Patterns of Urinary Pathogens in Female Outpatients. North American journal of medical sciences, 4(4), 163. https://doi.org/10.4103/1947-2714.94940

Sohail, M., Khurshid, M., Saleem, H. G. M., Javed, H., \& Khan, A. A. (2015). Characteristics and Antibiotic Resistance of Urinary Tract Pathogens Isolated from Punjab, Pakistan. Jundishapur journal of microbiology, 8(7). https://doi.org/10.5812/jjm.19272v2

Sood, S., Malhotra, M., Das, B., \& Kapil, A. (2008). Enterococcal Infections \& Antimicrobial Resistance. Indian Journal of Medical Research, 128(2), 111.

\section{Copyrights}

Copyright for this article is retained by the author(s), with first publication rights granted to the journal.

This is an open-access article distributed under the terms and conditions of the Creative Commons Attribution license (http://creativecommons.org/licenses/by/4.0/). 